\title{
Hemispheric Asymmetry of Auditory Mismatch Negativity Elicited by Spectral and Temporal Deviants: A Magnetoencephalographic Study
}

\author{
Hidehiko Okamoto $\cdot$ Ryusuke Kakigi
}

Received: 22 October 2013/Accepted: 16 December 2013/Published online: 24 December 2013

(C) The Author(s) 2013. This article is published with open access at Springerlink.com

\begin{abstract}
One of the major challenges in human brain science is the functional hemispheric asymmetry of auditory processing. Behavioral and neurophysiological studies have demonstrated that speech processing is dominantly handled in the left hemisphere, whereas music processing dominantly occurs in the right. Using magnetoencephalography, we measured the auditory mismatch negativity elicited by band-pass filtered click-trains, which deviated from frequently presented standard sound signals in a spectral or temporal domain. The results showed that spectral and temporal deviants were dominantly processed in the right and left hemispheres, respectively. Hemispheric asymmetry was not limited to high-level cognitive processes, but also originated from the pre-attentive neural processing stage represented by mismatch negativity.
\end{abstract}

Keywords Auditory evoked response $\cdot$ Hemispheric laterality $\cdot$ Magnetoencephalography (MEG) $\cdot$ Mismatch negativity (MMNm)

\section{Abbreviations \\ ANOVA Analysis of variance \\ MEG Magnetoencephalography \\ MMN Mismatch negativity}

This is one of several papers published together in Brain Topography on the "Special Issue: Auditory Cortex 2012".

Electronic supplementary material The online version of this article (doi:10.1007/s10548-013-0347-1) contains supplementary material, which is available to authorized users.

H. Okamoto $(\bowtie) \cdot$ R. Kakigi

Department of Integrative Physiology, National Institute for

Physiological Sciences, 38 Nishigo-Naka, Myodaiji,

Okazaki 444-8585, Japan

e-mail: hokamoto@nips.ac.jp $\begin{array}{ll}\text { SD } & \text { Spectral deviant } \\ \text { TD } & \text { Temporal deviant } \\ \text { TS } & \text { Test stimulus }\end{array}$

\section{Introduction}

Functional hemispheric asymmetry in the human brain has been investigated since the late nineteenth century (Wernicke 1874; Broca 1861). In addition to the classical behavioral observations of neurological disorder patients, recent neuroimaging techniques have made it possible to investigate conscious healthy human brains, and have revealed left hemispheric dominance for speech processing and right hemispheric dominance for music processing (Zatorre et al. 1994, 2002; Griffiths et al. 1999; Belin et al. 2000; Eulitz et al. 1995; Szymanski et al. 2001; Alho et al. 1998). However, functional hemispheric asymmetry in the human brain may not be limited to high-level cognitive neural processes, but may start from the lower neural processing level of basic acoustic features (e.g. frequency, interval, duration, and intensity).

Natural sounds have specific spectral distributions that change over time according to specific temporal sequences. Both spectral and temporal sound features have been shown to play an important role in the perception of natural sounds (Moore 2003); however, the importance of these features appears to differ between sound types, with spectral processing being of particular importance for music perception (Vos and Troost 1989; Warrier and Zatorre 2002) and temporal cues being essential for speech perception (Shannon et al. 1995; Drullman et al. 1994a, b). Recent functional magnetic resonance imaging (Jamison et al. 2006), positron emission tomography (Zatorre and Belin 2001), and magnetoencephalography (MEG) 
(Okamoto et al. 2009) studies have demonstrated using artificial basic auditory stimuli (e.g. pure tones and pulsetrains) that temporal changes are dominantly processed in the left hemisphere, whereas spectral changes are dominantly processed in the right. The well-known functional human hemispheric asymmetry observed for speech and music processing may not be limited to conscious highlevel cognitive processes, but may be at least partially related to the pre-attentive processing of low-level acoustic features.

Mismatch negativity (MMN) and its magnetic counterpart MMNm are auditory evoked components that reflect the cortical pre-attentive discrimination of auditory stimuli as well as auditory memory traces (Näätänen et al. 1978, 2007; Kujala et al. 2007). MMN(m) is elicited by violations of regularities in sound streams and can be recorded without any motor or other response and can even be obtained from inattentive patients and infants. Previous studies have shown that $\mathrm{MMN}(\mathrm{m})$ elicited by speech sounds was significantly lateralized to the left hemisphere (Alho et al. 1998), whereas MMN(m) elicited by musical notes was dominantly processed in the right hemisphere (Lappe et al. 2013; Tervaniemi et al. 1999). However, whether the hemispheric asymmetries of the pre-attentive $\mathrm{MMN}(\mathrm{m})$ are limited to meaningful auditory stimuli (e.g. speech and music) or originate from the basic spectral and temporal sound features of these sound stimuli remains unknown.

Therefore, the aim of the present study was to investigate the hemispheric laterality of MMNm evoked by spectral versus temporal sound deviants that do not convey specific phonological, grammatical, or musical information. In order to exclude the possibility that the laterality of the MMNm originated from the sound stimulus itself, we counter-balanced total auditory inputs identical between spectral and temporal deviant conditions. The results of the present study provide a new insight into how the left and right hemispheres pre-attentively deal with the spectral and temporal features of natural sound signals.

\section{Materials and Methods}

\section{Subjects}

Thirteen healthy subjects participated in this study (five females; mean \pm standard deviation: $32.1 \pm 6.2$ years). All participants had normal hearing, had no history of psychological or neurological disorders, and were unambiguously right-handed [assessed via the Japanese version of "Edinburgh Handedness Inventory" (Oldfield 1971)]. All participants were fully informed about the study and gave written informed consent for their participation in accordance with the procedures approved by the Ethics Commission of the National Institute for Physiological Sciences, Okazaki, Japan. The study conformed to the Code of Ethics of the World Medical Association (Declaration of Helsinki).

\section{Stimuli and Experimental Design}

The experimental design is schematically represented in Fig. 1. The test stimulus (TS) was either a $30 \mathrm{~Hz}$ (TS30) or $60 \mathrm{~Hz}$ (TS60) click-train, which was one-octave band-pass filtered either between 500 and 1,000 Hz (TS30_Low (Supplementary Audio 1S) and TS60_Low (Supplementary Audio 2S)) or between 1,000 and 2,000 Hz [TS30_High (Supplementary Audio 3S) and TS60_High (Supplementary Audio 4S)]. The TS had a duration of $330 \mathrm{~ms}$ and the sound onset asynchrony between the TS was $1,300 \mathrm{~ms}$. One of the TS were presented as standard stimuli with $70 \%$ probability pseudo-randomly intermixed with spectral deviants (SD: $15 \%$ probability) and temporal deviants (TD: $15 \%$ probability) in an oddball sequence as demonstrated in Fig. 1. In case of SD, band-pass filter settings changed from the standard stimulus, while the type of the click-train remained identical (standards and SD: TS30_Low and TS30_High, TS30_High and TS30_Low, TS60_Low and TS60_High, TS60_High and TS60_Low). On the other hand, in case of TD, the filter settings remained identical, while the type of click-train changed from the standard sound stimulus (standards and TD: TS30_Low and TS60_Low, TS30_High and TS60_High, TS60_Low and TS30_Low, TS60_High and TS30_High). More than two standard stimuli were presented before a deviant stimulus (SD or TD). Each MEG session consisted of four blocks. Each block contained four sub-blocks that pseudo-randomly adopted TS30_Low, TS30_High, TS60_Low, and TS60_High as the standard TS, respectively. Consequently, all TS types were presented with a probability of $25 \%$ in one block. Each sub-block had 21 SD, 21 TD, and 98 standard stimuli, resulting in a total number of 336 trials for each deviant stimulus and 1,568 trials for the standard condition. All sounds were diotically presented through plastic tubes $1.5 \mathrm{~m}$ in length and earpieces fitted to the subject's ears. Before starting an MEG measurement, each subject's hearing threshold for TS30_Low was individually determined for each ear. During the MEG recording session, TS30_Low was presented at an intensity of $60 \mathrm{~dB}$ above the individual sensation level, and other TS were adjusted to have power identical to TS30_Low. In order to keep the test subjects alert and distracted from the auditory signals, a self-chosen silent movie with captions was presented during the MEG recordings. Questions regarding the content of the movie were asked at the end of the measurement to ensure that the subjects had watched the movie. 
Fig. 1 Schematic depiction of the sound stimulation. Standard test stimuli $(70 \%)$ were presented together with spectral deviants (SD: $15 \%$ ) and temporal deviants (TD: $15 \%$ ) within an oddball paradigm

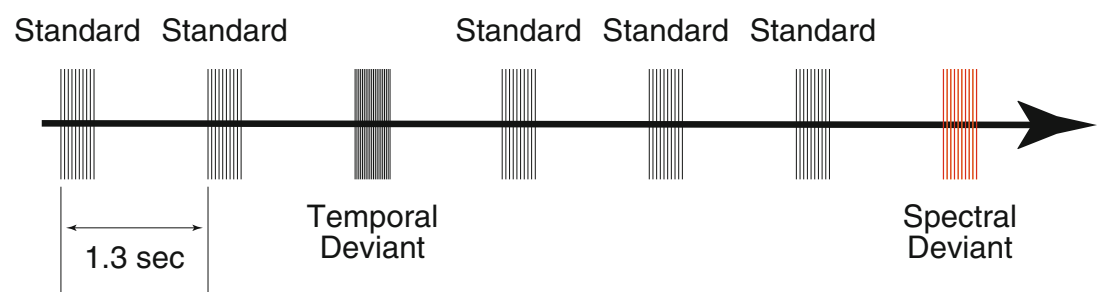

Data Acquisition and Analysis

Auditory evoked fields were recorded with a helmet-shaped, 306-channels MEG system (Vector-view, ELEKTA, Neuromag, Helsinki, Finland) with 102 identical triple sensor elements located in a silent, magnetically shielded room. We analyzed the MEG signals recorded by 204 planar-type gradiometers, detecting the largest signals over the corresponding cerebral sources. Signals were passed through a $0.03-200 \mathrm{~Hz}$ band-pass filter and digitized at $600 \mathrm{~Hz}$. The magnetic fields evoked by TS were selectively averaged for each condition (standard, SD, and TD) including pre- and post-stimulus intervals $(-100$ to $600 \mathrm{~ms}$ ). In the present study, TS onset (latency $=0 \mathrm{~ms}$ ) was defined when the first click of the TS reached the eardrum simulated by an artificial ear (Type 4157, Brüel \& Kjær Sound and Vibration Measurement, Nærum, Denmark). Subjects were instructed not to move their heads during the recordings and their compliance was monitored through a video camera by the experimenter. In order to improve the signal-to-noise ratio of the auditory evoked magnetic responses, epochs containing amplitude changes greater than $2.7 \mathrm{pT}$ within the pre- and post-stimulus intervals $(-100$ to $600 \mathrm{~ms})$ were automatically discarded as artifact-contaminated epochs. After artifact rejection, epochs were averaged for each condition (standard, SD, and TD), regardless of the sound types (TS30_Low, TS30_High, TS60_Low, and TS60_High). To analyze the MMNm component, which is elicited by deviant auditory signals (Näätänen et al. 2007; Alho 1995), the averaged auditory evoked fields in each condition (SD, TD, and standard stimuli) were $1-30 \mathrm{~Hz}$ band-pass filtered in order to extract the transient evoked responses, and the baseline was corrected relative to the $100 \mathrm{~ms}$ pre-stimulus interval. Thereafter, in order to obtain the MMNm waveforms elicited by SD (MMNm_SD) and TD (MMNm_TD), the auditory evoked fields elicited by the standard TS were subtracted from those elicited by SD and TD. The onset of SD matched with the first click of the TS (latency $=0 \mathrm{~ms}$ ), whereas TD did not occur at the first click of the TS. When $30 \mathrm{~Hz}$ click trains (TS30_Low or TS30_High) were used as the standard TS, the temporal deviant occurred at the presentation of the second click of TS60_Low or TS60_High (latency $=16.7 \mathrm{~ms}$ ). When $60 \mathrm{~Hz}$ click trains (TS60_Low or TS60_High) were used as the standard TS, the onset of TD could be the timing of the missing second click of the standard stimuli (latency $=16.7 \mathrm{~ms}$ ). Therefore, after obtaining the subtracted magnetic waveforms (MMNm_SD and MMNm_TD) the latency of MMNm_TD was offset by a reduction in $16.7 \mathrm{~ms}$ and was then used for the subsequent statistical analysis.

In order to investigate differences in the magnetic sensors, the time courses of the root-mean-square (RMS) amplitudes of the subtracted magnetic fields (MMNm_SD or MMNm_TD) were calculated by using all of the leftside (96 sensors) or right-side (96 sensors) planar-type gradiometers in each subject. The most prominent RMS peak in each hemisphere ranging from 100 to $250 \mathrm{~ms}$ after the sound onset was defined as the MMNm response in each subject. The mean RMS value within the $10 \mathrm{~ms}$ time window around the RMS peak in each condition, each side, and each subject was used in statistical analysis. The mean RMS amplitudes and latencies of the MMNm responses were evaluated separately by means of repeated-measures analyses of variance (ANOVA) using the two factors DEVIANT_CONDITION [spectral deviant (SD) vs. temporal deviant (TD)] and HEMISPHERE (left vs. right).

The estimated single dipole source strength was shown to be modulated easily by the depth of the estimated location (Hillebrand and Barnes 2002). We could obtain reliable source strengths using identical source locations and orientations between conditions. In order to improve the signal-to-noise ratio, we averaged MMNm_SD and MMNm_TD in each subject and used the averaged magnetic waveforms to estimate the single equivalent current dipoles reflecting the MMNm response. The peak MMNm response was initially identified as the maximal RMS value of the global field power between 100 and $250 \mathrm{~ms}$ after TS onset. A $10 \mathrm{~ms}$ interval around the MMNm peak latency was selected, and the source locations and orientations were estimated using single equivalent current dipole modeling (one dipole per hemisphere) for each subject individually (BESA Research 5.3.7, BESA GmbH, Germany). We calculated the two equivalent current dipoles (one dipole per hemisphere) simultaneously by using all whole-head planar-type gradiometers (204 channels) for the MMNm source estimation. Dipole estimation was not successful in one subject, which reduced the number of subjects to $N=12$. The goodness-of-fit for the MMNm dipoles of the remaining 12 subjects was more than $80 \%$ (mean \pm standard deviation: $89.0 \pm 3.0$ ). The estimated 
sources, which were fixed in location and orientation for each hemisphere of each subject, served as a spatial filter (Tesche et al. 1995) to calculate the source strength for each condition (SD and TD) and in each hemisphere (left and right) of each subject. The mean source strength within the $10 \mathrm{~ms}$ time window around the peak MMNm latency was used for further statistical analysis of the MMNm. In order to evaluate the effects of the deviant type and hemisphere, the source strengths and latencies of the estimated equivalent current dipoles corresponding to the MMNm responses elicited by the deviant stimuli (SD and TD) in each hemisphere were evaluated separately via a repeated-measures ANOVA using the two factors DEVIANT_CONDITION (SD vs. TD) and HEMISPHERE (Left vs. Right).

\section{Results}

Twelve subjects (except for one excluded subject) underwent an adequate number of trials to obtain auditory evoked fields for each condition after the artifact rejection [mean \pm standard deviation: $\mathrm{SD}=332.7 \pm 3.6(99.0 \pm 1.1 \%)$, $\mathrm{TD}=$ $333.8 \pm 1.7(99.3 \pm 0.5 \%)$, standard stimuli $=1556.2 \pm$ $9.3(99.2 \pm 0.6 \%)]$. An example of individual magnetic field waveforms in each condition (SD, TD, and standard) and subtracted waveforms [MMNm_SD (SD-standard), MMNm_TD (TD-standard)] is shown in Fig. 2, which demonstrates the clear N1m-responses elicited by TS onset in the upper panels as well as MMNm-responses in the subtracted waveforms in the lower panels.

The calculated means of the RMS values of the auditory evoked fields for each condition (MMNm_SD and MMNm_TD) in each hemisphere averaged across 12 subjects are displayed in Fig. 3, in which the RMS waveforms elicited by TD were shifted $16.7 \mathrm{~ms}$ to the left-side in order to adjust the timing of the deviant sound onset. Clear MMNm responses were observed in both conditions and hemispheres. The RMS peaks in the MMNm_TD condition were later than those in the MMNm_SD condition in both hemispheres.

The mean RMS amplitudes and latencies of the MMNm responses averaged across 12 subjects for each condition in each hemisphere are presented in Fig. 4 with error bars denoting the $95 \%$ confidence intervals calculated by the means of bootstrap resampling tests (iteration $=100,000$ ). The repeated-measures ANOVA applied to the maximal RMS amplitudes of the MMNm responses in each hemisphere resulted in a significant main effect for DEVIANT_CONDITION $\left(\mathrm{F}_{(1,11)}=11.78, p<0.01\right)$, but not for HEMISPHERE $\left(\mathrm{F}_{(1,11)}=2.53, p=0.14\right)$. Additionally, a marginal trend toward significance was observed in the interaction between DEVIANT_CONDITION and HEMISPHERE $\left[\mathrm{F}_{(1,11)}=4.54, p=0.056\right]$. The repeated-measures ANOVA applied to the latencies of the maximal RMS amplitudes of the MMNm responses resulted in a
Fig. 2 Examples of individual magnetic waveforms. The upper panels represent the auditory evoked fields of one representative subject elicited by a spectral deviant (SD), b standard, and $\mathbf{c}$ temporal deviant (TD) sound stimuli. The lower panels show the magnetic waveforms obtained by the subtraction between a and b [d spectral mismatch negativity (MMNm_SD)] and between $\mathbf{c}$ and $\mathbf{b}$ [e temporal mismatch negativity (MMNm_TD)]

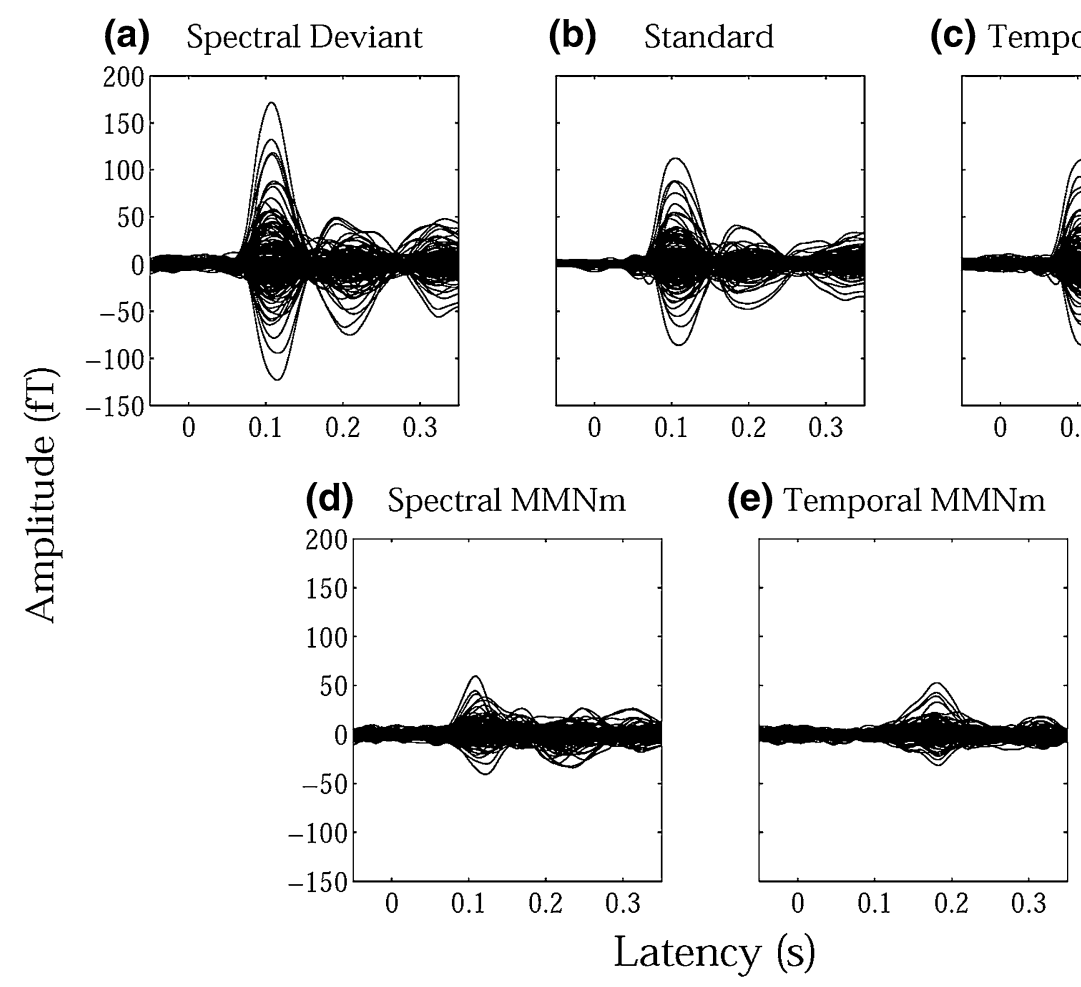




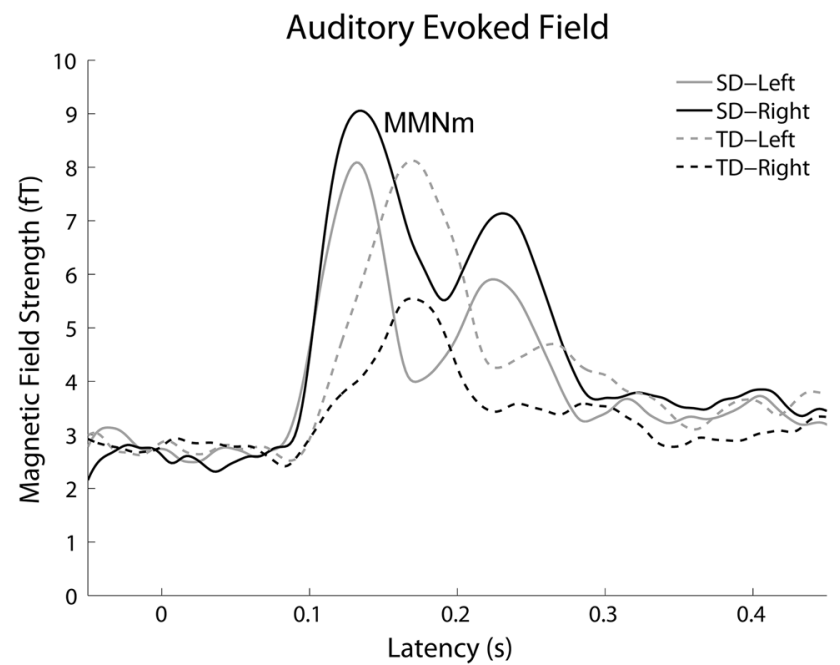

Fig. 3 Grand-averaged $(N=12)$ root-mean-square (RMS) values of the magnetic fields (left panel) and grand-averaged source strengths (right panel) of the mismatch negativity (MMNm) waveforms. Solid and dashed lines represent the spectral deviant (MMNm_SD) and the

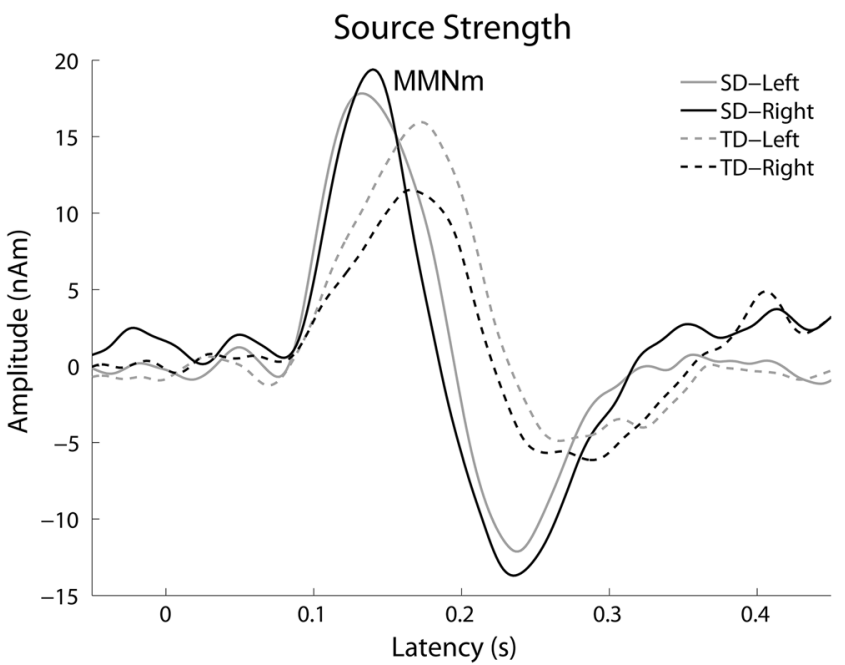

temporal deviant (MMNm_TD) conditions, respectively. Gray lines represent the left sensor (left panel) and left hemisphere (right panel) and black lines represent the right sensor (left panel) and right hemisphere (right panel)
Fig. 4 The left and right graphs display the mean root-meansquare (RMS) values and latencies of the magnetic fields corresponding to the mismatch negativity (MMNm) and mean MMNm source strengths and latencies with error bars denoting $95 \%$ confidence intervals, respectively. Filled bars denote the left sensor (LS: left panels) and left hemisphere (LH: right panels) responses and open bars denote the right sensor (RS: left panels) or right hemisphere (RH: right panels) responses
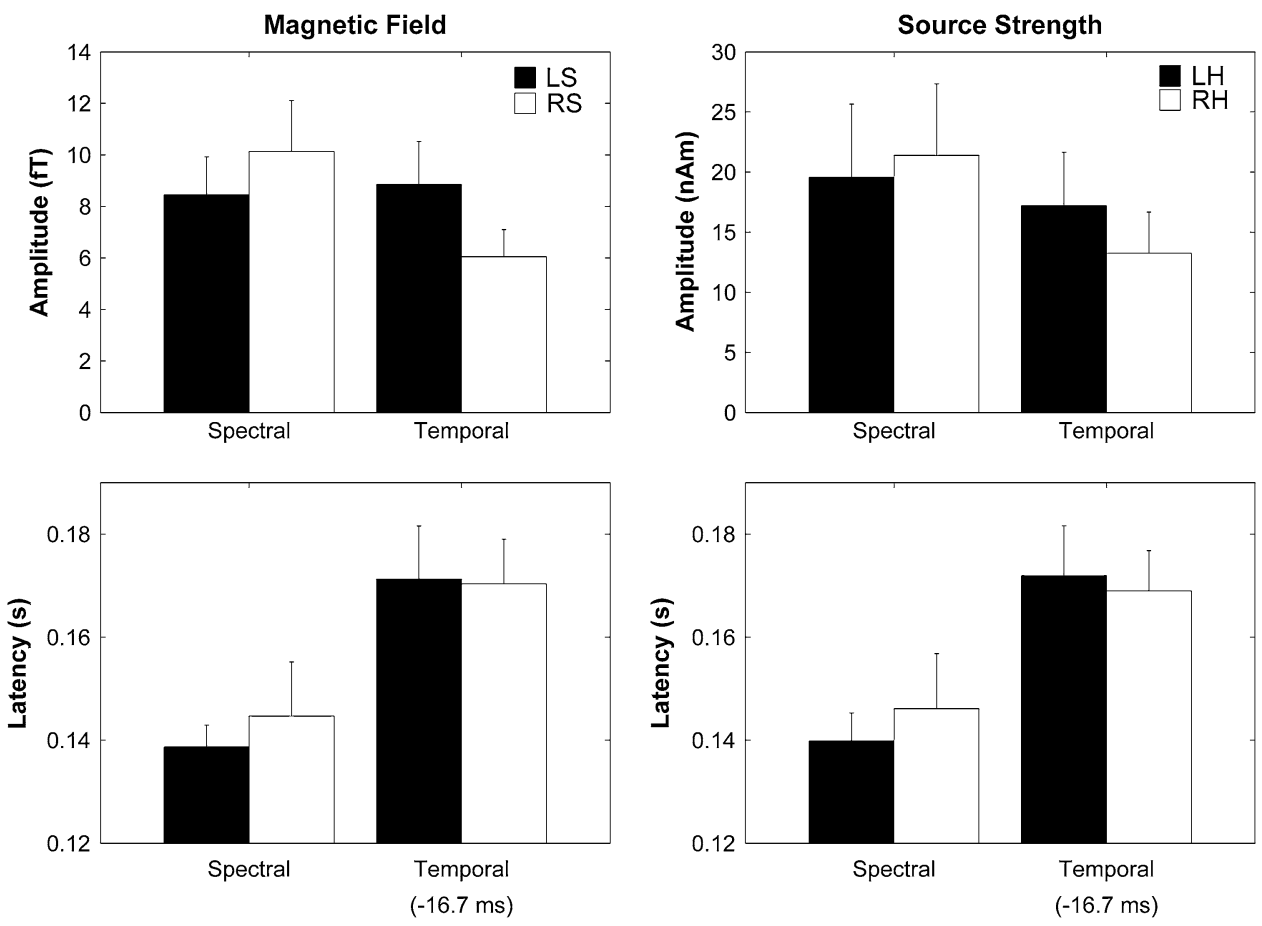

significant main effect for DEVIANT_CONDITION $\left[\mathrm{F}_{(1,11)}=36.30, p<0.001\right]$, but neither a significant main effect nor a significant interaction were observed [HEMI$\operatorname{SPHERE}\left[\mathrm{F}_{(1,11)}=0.33, p=0.58\right]$; DEVIANT_CONDITION $\times$ HEMISPHERE $\left[\mathrm{F}_{(1,11)}=0.78, p=0.40\right]$.

The calculated means of the MMNm source strength waveforms for each hemisphere averaged across 12 subjects are displayed in Fig. 3, in which MMNm source strength waveforms elicited by TD were shifted $16.7 \mathrm{~ms}$ to the leftside. Clear MMNm-responses ranging between 100 and
$200 \mathrm{~ms}$ were observed in both hemispheres after TS onset. The mean MMNm source strengths and latencies averaged across 12 subjects for each condition in each hemisphere are presented in Fig. 4 with error bars denoting the $95 \%$ confidence intervals calculated by means of bootstrap resampling tests (iteration $=100,000$ ). The repeated-measures ANOVA applied to the MMNm source strengths revealed a significant main effect for DEVIANT_CONDITION $\left[\mathrm{F}_{(1,11)}=6.44, p<0.03\right]$. Additionally, a significant interaction was observed between DEVIANT_CONDITION and 
HEMISPHERE $\left[\mathrm{F}_{(1,11)}=6.67, p<0.03\right]$, which indicated that the MMNm response elicited by SD was relatively larger in the right hemisphere, whereas the MMNm response elicited by TD was relatively larger in the left hemisphere. The repeated-measures ANOVA applied to the MMNm latencies revealed a significant main effect for DEVIANT_CONDITION $\left[\mathrm{F}_{(1,11)}=48.45, p<0.001\right]$, but no significant interaction between factors: MMNm_TD was significantly longer than that of MMNm_SD.

We also analyzed MMNm source strengths and latencies when the MMNm_TD was not shifted by $16.7 \mathrm{~ms}$ during the calculation. A repeated-measures ANOVA performed on the MMNm source strengths revealed a significant main effect for DEVIANT_CONDITION $\left[\mathrm{F}_{(1,11)}=6.29, p<0.03\right]$ and a significant interaction between DEVIANT_CONDITION and HEMISPHERE $\left[\mathrm{F}_{(1,11)}=6.87, p<0.03\right]$. A repeatedmeasures ANOVA performed on the MMNm latencies revealed a significant main effect for DEVIANT_CONDITION $\left[\mathrm{F}_{(1,11)}=125.3, p<0.001\right]$, but no significant interaction between factors.

\section{Discussion}

The results obtained in the present study clearly demonstrated a difference in the hemispheric laterality of MMNm amplitudes between SD and TD conditions. The amplitudes of MMNm evoked by SD (MMNm_SD) were relatively larger in the right, whereas those evoked by TD (MMNm_TD) were relatively larger in the left (Figs. 3, 4). No hemispheric difference was observed in the MMNm latency; however, the latencies of MMNm_TD were significantly longer than those of MMNm_SD in both the left and right hemispheres even when the onset time difference between SD and TD (16.7 ms) was considered. In contrast to previous studies (Alho et al. 1998; Shtyrov et al. 2000), which also investigated the hemispheric asymmetry of $\operatorname{MMN}(\mathrm{m})$, the total sound inputs were identical between SD and TD conditions in the present study. Therefore, the sound property itself cannot explain the obtained results; the deviation pattern (SD or TD) from the standard sound stream was solely responsible for the results obtained. We used band-pass filtered click-trains that did not convey specific meanings to ensure that hemispheric lateralization for pre-attentive human auditory processing, represented by $\mathrm{MMN}(\mathrm{m})$, was not limited to the complex waveforms from natural sound sources (e.g. human voice or musical instruments), but in part originated from early, low-level auditory neural processing dealing with basic sound characteristics, namely, spectral and temporal features (Zatorre and Belin 2001; Tallal et al. 1993; Poeppel 2003; Boemio et al. 2005).
It seems plausible that spectral and temporal sound information is differentially encoded into neural activity (Bendor and Wang 2007; Sakai et al. 2009). Spectral information is encoded into the maximal movement position of the basilar membrane in the cochlea. Therefore, in case of the SD condition, the groups of inner hair cells corresponding to SD sounds were different from those corresponding to standard sounds. In contrast, TD sounds had similar frequency characteristics to standard sound signals. Similar groups of inner hair cells on the tonotopic map in the cochlea are activated. In order to detect the TD sound signal, the central auditory system should analyze the temporal patterns of neural activity. The present results demonstrated that the MMNm latencies elicited by TD were significantly longer than those elicited by SD (Figs. 3, 4). First, we have to consider the timing of the SD and TD onsets. Theoretically, SD is detectable from the first click of the TS in the cochlea, whereas TD detection requires the second click of the $60 \mathrm{~Hz}$ band-pass filtered click trains deviated from the standard $30 \mathrm{~Hz}$ band-pass filtered clicks or the missing second click of the standard $60 \mathrm{~Hz}$ bandpass filtered click trains during presentation of the deviant $30 \mathrm{~Hz}$ band-pass filtered click trains to manifest in the central auditory system. Therefore, we first subtracted $16.7 \mathrm{~ms}$ from the MMNm_TD latency in order to compare it with the MMNm_SD latency. Even after this adjustment, MMNm_TD was significantly longer than MMNm_SD (Fig. 4), which suggested that different neural mechanisms contribute to the detection of spectral and temporal sound deviants. Neural encoding of the temporal patterns of auditory signals took longer and appeared to take place at a higher level of the auditory system than spectral coding. Previous MEG studies (Okamoto et al. 2009, 2012) also support this hypothesis by demonstrating that the temporal changes elicited significantly delayed auditory N1m responses, with a major deflection in the auditory evoked response having a latency of approximately $100 \mathrm{~ms}$ (Näätänen and Picton 1987), than those elicited by spectral changes.

Auditory MMNm is a pre-attentive automatic brain response elicited by any change in auditory stimulation (Näätänen et al. 2007). In the present study, we used bandpass filtered click trains that did not convey specific meaning and subjects were distracted from the auditory modality; therefore, it is less likely that subjects involuntarily processed and perceived the test sounds as musical or speech signals. The obtained results indicated that the hemispheric asymmetry of auditory processing in humans starts from the basic, pre-attentive auditory processing level. Moreover, sound inputs were completely counterbalanced between the SD and TD conditions. Therefore, the hemispheric asymmetry of the MMNm responses elicited by the SD and TD could not be explained solely by 
stimulus features. The lateralized memory traces of basic auditory processes in terms of spectral and temporal sound features appear to be responsible for the results obtained. Recent human neuroimaging studies revealed that the functional hemispheric asymmetry of auditory processing was not limited to complex sound signals conveying specific meaning and rules (e.g. music and speech), but originated from the basic auditory processing level, namely, the temporal integration window (Poeppel 2003; Belin et al. 1998; Zatorre and Belin 2001; Zatorre et al. 2002). It is important to quickly and precisely encode environmental sounds in daily life. However, because of the trade-off between temporal and spectral analysis precision [Acoustic uncertainty principle; (Joos 1948; Zatorre et al. 2002)], it is impossible to achieve high spectral and high temporal sound analyses at the same time using one temporal integration window. A short temporal integration window leads to high temporal resolution, but relatively low spectral resolution of the sound analyses. On the other hand, a long temporal window leads to high spectral resolution, but relatively low temporal resolution of the sound analyses. Therefore, it seems plausible that the human auditory cortices in the left and right hemispheres adopt different integration time windows instead of applying one specific temporal integration time window in both hemispheres. Belin et al. (1998) and Poeppel (2003) hypothesized that the left hemisphere applied a shorter temporal integration window, resulting in a better temporal resolution capability, and the right hemisphere applied a longer temporal integration window, resulting in a better spectral resolution capability. In the present study, the longer temporal integration window with higher spectral resolution in the right hemisphere appears to have dominantly contributed to detecting spectrally deviated sound signals and resulted in relatively larger MMNm_SD amplitudes in the right hemisphere. In contrast, the shorter temporal integration window with high temporal resolution in the left hemisphere appears to have dominantly processed temporally deviated sound signals and resulted in relatively larger MMNm_TD amplitudes in the left hemisphere.

The MMNm amplitudes and latencies obtained in the sensor space and source space exhibited similar patterns (Figs. 3, 4): the MMNm_SD and MMNm_TD amplitudes were larger in the right and left hemispheres, respectively. However, the ANOVA examining MMNm amplitudes resulted in a significant interaction between DEVIANT_CONDITION and HEMISPHERE in the source space data $\left[\mathrm{F}_{(1,11)}=6.67\right.$, $p<0.03$ ], but only a marginal trend toward significance was observed in the sensor space data $\left[\mathrm{F}_{(1,11)}=4.54, p=0.056\right]$. The main reason for this inconsistency may be that the neural sources in one hemisphere could influence the evoked magnetic fields in the contra-lateral magnetic sensors. Moreover, head sizes and head positions differed between subjects and the central sulcus of the subjects could shift from the center of the MEG dewar. Therefore, these factors may have led to a less robust statistical outcome in the RMS amplitudes of the MMNm responses than the MMNm source strengths.

In conclusion, using carefully constructed auditory stimuli that were counter-balanced between conditions and had clear time-locked onsets of SD and TD, the present study clearly demonstrated that neural processing dealing with spectrally deviated sounds were relatively dominant in the right hemisphere while those dealing with temporally deviated sounds were relatively dominant in the left hemisphere. These results strongly support the hypothesis that the human brain adopts asymmetric memory traces of basic spectral and temporal sound features in the left and right hemispheres in order to improve the detection of deviant sound signals.

Acknowledgments We thank Y. Takeshima for technical help and our test subjects for their diligent collaboration. This study was supported by the "Japan Society for the Promotion of Science for Young Scientists (23689070)", "Strategic Research Program for Brain Sciences (Development of biomarker candidates for social behavior)", and "Sound Technology Promotion Foundation".

Conflict of interest The authors have declared that no competing interests exist.

Open Access This article is distributed under the terms of the Creative Commons Attribution License which permits any use, distribution, and reproduction in any medium, provided the original author(s) and the source are credited.

\section{References}

Alho K (1995) Cerebral generators of mismatch negativity (MMN) and its magnetic counterpart (MMNm) elicited by sound changes. Ear Hear 16:38-51

Alho K, Connolly JF, Cheour M, Lehtokoski A, Huotilainen M, Virtanen J, Aulanko R, Ilmoniemi RJ (1998) Hemispheric lateralization in preattentive processing of speech sounds. Neurosci Lett 258:9-12

Belin P, Zilbovicius M, Crozier S, Thivard L, Fontaine A (1998) Lateralization of speech and auditory temporal processing. J Cogn Neurosci 10:536-540

Belin P, Zatorre RJ, Lafaille P, Ahad P, Pike B (2000) Voice-selective areas in human auditory cortex. Nature 403:309-312

Bendor D, Wang XQ (2007) Differential neural coding of acoustic flutter within primate auditory cortex. Nat Neurosci 10:763-771

Boemio A, Fromm S, Braun A, Poeppel D (2005) Hierarchical and asymmetric temporal sensitivity in human auditory cortices. Nat Neurosci 8:389-395

Broca P (1861) Remarques sur le siége de la faculté du langage articulé suivies d'une observation d'aphémie (perte de la parole). Bull Soc Anat 6:330-357

Drullman R, Festen JM, Plomp R (1994a) Effect of reducing slow temporal modulations on speech reception. J Acoust Soc Am 95:2670-2680

Drullman R, Festen JM, Plomp R (1994b) Effect of temporal envelope smearing on speech reception. J Acoust Soc Am 95:1053-1064 
Eulitz C, Diesch E, Pantev C, Hampson S, Elbert T (1995) Magnetic and electric brain activity evoked by the processing of tone and vowel stimuli. J Neurosci 15:2748-2755

Griffiths TD, Johnsrude I, Dean JL, Green GG (1999) A common neural substrate for the analysis of pitch and duration pattern in segmented sound? Neuroreport 10:3825-3830

Hillebrand A, Barnes GR (2002) A quantitative assessment of the sensitivity of whole-head MEG to activity in the adult human cortex. Neuroimage 16:638-650

Jamison HL, Watkins KE, Bishop DV, Matthews PM (2006) Hemispheric specialization for processing auditory nonspeech stimuli. Cereb Cortex 16:1266-1275

Joos M (1948) Acoustic phonetics. Language monograph, vol 23. Linguistic Society of America, Baltimore

Kujala T, Tervaniemi M, Schroger E (2007) The mismatch negativity in cognitive and clinical neuroscience: theoretical and methodological considerations. Biol Psychol 74:1-19

Lappe C, Steinsträter O, Pantev C (2013) A beamformer analysis of MEG data reveals frontal generators of the musically elicited mismatch negativity. PLoS One 8:e61296

Moore BCJ (2003) An introduction to the psychology of hearing. Academic Press, Boston

Näätänen R, Picton T (1987) The N1 wave of the human electric and magnetic response to sound: a review and an analysis of the component structure. Psychophysiology 24:375-425

Näätänen R, Gaillard AW, Mäntysalo S (1978) Early selectiveattention effect on evoked potential reinterpreted. Acta Psychol 42:313-329

Näätänen R, Paavilainen P, Rinne T, Alho K (2007) The mismatch negativity (MMN) in basic research of central auditory processing: a review. Clin Neurophysiol 118:2544-2590

Okamoto H, Stracke H, Draganova R, Pantev C (2009) Hemispheric asymmetry of auditory evoked fields elicited by spectral versus temporal stimulus change. Cereb Cortex 19:2290-2297

Okamoto H, Teismann H, Kakigi R, Pantev C (2012) Auditory evoked fields elicited by spectral, temporal, and spectraltemporal changes in human cerebral cortex. Front Psychol 3:149

Oldfield RC (1971) The assessment and analysis of handedness: the Edinburgh inventory. Neuropsychologia 9:97-113

Poeppel D (2003) The analysis of speech in different temporal integration windows: cerebral lateralization as 'asymmetric sampling in time'. Speech Commun 41:245-255
Sakai M, Chimoto S, Qin L, Sato Y (2009) Differential representation of spectral and temporal information by primary auditory cortex neurons in awake cats: relevance to auditory scene analysis. Brain Res Cogn Brain Res 1265:80-92

Shannon RV, Zeng FG, Kamath V, Wygonski J, Ekelid M (1995) Speech recognition with primarily temporal cues. Science 270:303-304

Shtyrov Y, Kujala T, Palva S, Ilmoniemi RJ, Naatanen R (2000) Discrimination of speech and of complex nonspeech sounds of different temporal structure in the left and right cerebral hemispheres. Neuroimage 12:657-663

Szymanski MD, Perry DW, Gage NM, Rowley HA, Walker J, Berger MS, Roberts TP (2001) Magnetic source imaging of late evoked field responses to vowels: toward an assessment of hemispheric dominance for language. J Neurosurg 94:445-453

Tallal P, Miller S, Fitch RH (1993) Neurobiological basis of speech: a case for the preeminence of temporal processing. Ann N Y Acad Sci 682:27-47

Tervaniemi M, Kujala A, Alho K, Virtanen J, Ilmoniemi RJ, Näätänen R (1999) Functional specialization of the human auditory cortex in processing phonetic and musical sounds: a magnetoencephalographic (MEG) study. Neuroimage 9:330-336

Tesche CD, Uusitalo MA, Ilmoniemi RJ, Huotilainen M, Kajola M, Salonen O (1995) Signal-space projections of meg data characterize both distributed and well-localized neuronal sources. Electroencephalogr Clin Neurophysiol Suppl 95:189-200

Vos PG, Troost JM (1989) Ascending and descending melodic intervals-statistical findings and their perceptual relevance. Music Percept 6:383-396

Warrier CM, Zatorre RJ (2002) Influence of tonal context and timbral variation on perception of pitch. Percept Psychophys 64:198-207

Wernicke C (1874) Symptomenkomplex. Eine psychologische Studie auf anatomischer Basis. Cohn und Weigert, Breslau

Zatorre RJ, Belin P (2001) Spectral and temporal processing in human auditory cortex. Cereb Cortex 11:946-953

Zatorre RJ, Evans AC, Meyer E (1994) Neural mechanisms underlying melodic perception and memory for pitch. J Neurosci 14:1908-1919

Zatorre RJ, Belin P, Penhune VB (2002) Structure and function of auditory cortex: music and speech. Trends Cogn Sci 6:37-46 